\title{
Effective computation of $\operatorname{Tor}_{k}(M, N)$
}

\author{
Socorro García Román * Manuel García Román
}

November 9, 2018

\begin{abstract}
An effective method to compute a presentation of $\operatorname{Tor}_{k}(M, N)$ for modules on a not necessarily commutative algebra is proposed.
\end{abstract}

\section{Introduction}

Unlike $\operatorname{Ext}_{k}(M, N)$ (see 4 for an algorithm when the base ring $R$ is a commutative algebra and [1] when $R$ is a PBW algebra), there are not known effective methods to compute $\operatorname{Tor}_{k}(M, N)$ for a pair of $R$-modules $M$ and $N$.

In this note, we propose an algorithm to compute a presentation of $\operatorname{Tor}_{k}(M, N)$ when $R$ is a Poincaré-Birkhoff-Witt algebra (PBW algebra for short; see 11, 2, et.al.] for a definition and examples, including the Weyl algebras, universal enveloping algebras of Lie algebras, the quantum plane, algebras of quantum matrices and other iterated Ore extensions, etc.). Since in general $\operatorname{Tor}_{k}(M, N)$ is just an abelian group when $M$ and $N$ are left $R$ modules, we ask for a two-sided structure on $M$. When $M$ is an $R$-bimodule then $\operatorname{Tor}_{k}(M, N)$ is a left $R$-module. We show that if, in addition, $M$ is finitely generated and $N$ is a finitely generated left $R$-module, then effective techniques involving Gr̈obner bases may be used to compute $\operatorname{Tor}_{k}(M, N)$ for any $k \geq 0$.

Besides the standard algorithm to compute (left) syzygies, the ingredients of our method, are

- an algorithm for computing a free resolution of the left $R$-module $N$ given a finite system of generators of $N$. Such an algorithm may be found in 2];

*Supported by DGUI, Consejería de Educación - Gobierno de Canarias 
- a finite presentation of the $R$-bimodule $M$. Using the syzygy bimodule described in [3], we show below a method for computing such a presentation when $M$ is a centralizing $R$-bimodule and a finite system of two-sided generators of $M$ is given;

- theorem 2.2

Our algorithm, described in detail in 2.3, follows the lines of [1].

\section{Preliminaries}

This section is devoted to fixing notation and describing some isomorphisms which will be used later.

Let $M$ be an $R$-bimodule.

For all $s \geq 1$, the map $\alpha: M^{s} \longrightarrow M \otimes_{R} R^{s} ; \alpha\left(f_{1}, \ldots, f_{s}\right)=\sum_{i=1}^{s} f_{i} \otimes e_{i}$, where $\left\{e_{1}, . ., e_{s}\right\}$ is the canonical basis of $R^{s}$, is an isomorphism of left $R$ modules.

Indeed, $\alpha$ is the composition of the isomorphisms

$$
\begin{aligned}
& M^{s} \quad \longrightarrow \quad\left(M \otimes_{R} R\right)^{s} \quad \longrightarrow M \otimes_{R} R^{s} \\
& \left(f_{1}, \ldots, f_{s}\right) \mapsto\left(f_{1} \otimes 1, \ldots, f_{s} \otimes 1\right) \mapsto \sum_{i=1}^{s} f_{i} \otimes e_{i} .
\end{aligned}
$$

On the other hand, if $A$ is a subbimodule of $R^{m}$ and $B$ is a left submodule of $R^{s}$, then

$$
\begin{aligned}
& \beta:\left(R^{m} \otimes_{R} R^{s}\right) / T \quad \longrightarrow\left(R^{m} / A\right) \otimes_{R}\left(R^{s} / B\right) \\
& (f \otimes g)+T \mapsto \quad(f+A) \otimes(g+B),
\end{aligned}
$$

where $T=R^{m} \otimes B+A \otimes_{R} R^{s}$, is an isomorphism of left $R$-modules.

Furthermore, if $\left\{a_{1}, \ldots, a_{r}\right\}$ is a generator system of $A$ such that $A=$ $R a_{1}+\cdots+R a_{r}=a_{1} R+\cdots+a_{r} R$ and $\left\{b_{1}, \ldots, b_{t}\right\}$ is a generator system of $B$ as a left $R$-module, then

$$
\left\{a_{i} \otimes b_{j} / 1 \leq i \leq r, 1 \leq j \leq t\right\}
$$

is a generator system of $A \otimes_{R} B$ as a left $R$-module, since for all $a=$ 


$$
\begin{aligned}
& \sum_{l=l}^{r} p_{l} a_{l} \in A \text { and } b=\sum_{j=1}^{t} p_{j}^{\prime} b_{j} \in B \text { with } p_{l}, p_{j}^{\prime} \in R, \\
& a \otimes b=\sum_{j, l} p_{l} a_{l} \otimes p_{j}^{\prime} b_{j} \\
&=\sum_{j, l} p_{l}\left(a_{l} p_{j}^{\prime}\right) \otimes b_{j} \\
&=\sum_{j, l} p_{l}\left(\sum_{i=1}^{r} p_{i}^{j l} a_{i}\right) \otimes b_{j} \\
&=\sum_{i, j, l} p_{l} p^{j l} a_{i} \otimes b_{j} .
\end{aligned}
$$

¿From here on, let $0 \rightarrow L \rightarrow R^{m} \stackrel{p_{M}}{\rightarrow} M \rightarrow 0$ be a finite presentation of the $R$-bimodule $M$, and let $H=\left\{h_{1}, \ldots, h_{r}\right\} \subseteq R^{m}$ be a two-sided generator system of $L$ in such a way that $L=R H=H R$ (e.g., $H$ is a two-sided Gröbner basis of $L$ ).

Remark 1.1. If $M$ is a centralizing bimodule, such a presentation may be computed as follows. Recall that the $R$-bimodule $M$ is said to be centralizing if $M$ is generated as a left $R$-module (or equivalently, as a right $R$-module) by its centralizer

$$
\operatorname{Cen}_{R} M=\{m \in M ; r m=m r, \forall r \in R\} .
$$

Let $R$ be a PBW algebra and $M$ an $R$-bimodule. In [3] we define the syzygy bimodule of $\left\{m_{1}, \ldots, m_{s}\right\} \subseteq M$ as the kernel of the morphism of left $R^{\text {env }}$ modules $\left(R^{\text {env }}\right)^{s} \longrightarrow M ; \quad e_{i} \longmapsto m_{i}$, where $R^{\text {env }}$ is the enveloping algebra $R \otimes R^{\mathrm{op}}$. We also provide an algorithm to compute the syzygy bimodule of $\left\{m_{1}, \ldots, m_{s}\right\}$.

If the $R$-bimodule $M$ is centralizing, say generated by $\left\{m_{1}, \ldots, m_{s}\right\} \in$ $\operatorname{Cen}_{R} M$, then the syzygy bimodule is $\left(\mathfrak{m}^{s}\right)^{-1} L$, where $\mathfrak{m}^{s}:\left(R^{\text {env }}\right)^{s} \longrightarrow R^{s}$ is given by $\mathfrak{m}(f \otimes g)=f g$ and $L$ is the kernel of the epimorphism $p_{M}: R^{s} \longrightarrow$ $M$. In that case, if $G$ is a (left) Gröbner basis of the syzygy bimodule, then $H=\mathfrak{m}^{s}(G) \backslash\{0\}$ is a two-sided Gröbner basis of $L$ (cf. 3, thm. 7]).

If $N$ is a left $R$-module and $0 \rightarrow B \rightarrow R^{s} \rightarrow N \rightarrow 0$ is a presentation of $N$, then the map

$$
\begin{aligned}
& \gamma: R^{m s} / \alpha^{-1}(T) \longrightarrow M \otimes_{R} N \\
& e_{i j}+\alpha^{-1}(T) \longmapsto p_{M}\left(e_{j}^{\prime}\right) \otimes p_{N}\left(e_{i}\right)
\end{aligned}
$$


is an isomorphism of left $R$-modules, where $T=R^{m} \otimes_{R} B+L \otimes_{R} R^{s}$, and $\left\{e_{1}, \ldots, e_{s}\right\}$, resp. $\left\{e_{1}^{\prime}, \ldots, e_{m}^{\prime}\right\}$, resp. $\left\{e_{11}, \ldots, e_{1 m}, \ldots, e_{s 1}, \ldots, e_{s m}\right\}$ are the canonical bases of $R^{s}$, resp. $R^{m}$, resp. $R^{m s}=\left(R^{m}\right)^{s}$, being $e_{i j}=\left(0_{R^{m}}, \ldots, e_{j}^{\prime}\right.$ $\left., \ldots, 0_{R^{m}}\right)$.

Indeed, $\gamma$ is the composition of isomorphisms

$$
R^{m s} / \alpha^{-1}(T) \stackrel{\bar{\alpha}}{\longrightarrow}\left(R^{m} \otimes_{R} R^{s}\right) / T \stackrel{\beta}{\longrightarrow}\left(R^{m} / L\right) \otimes_{R}\left(R^{s} / B\right) \stackrel{\bar{p}_{M} \otimes \bar{p}_{N}}{\longrightarrow} M \otimes_{R} N
$$

where $\bar{\alpha}$, resp. $\bar{p}_{M}$, resp. $\bar{p}_{N}$ is obtained by factoring $\alpha: R^{m s} \longrightarrow\left(R^{m} \otimes_{R}\right.$ $\left.R^{s}\right) / T$, resp. $p_{M}: R^{m} \longrightarrow M$, resp. $p_{N}: R^{s} \longrightarrow N$ through the quotient.

Moreover, if $\left\{g_{1}, \ldots, g_{t}\right\}$ is a generator system of $B$ as a left $R$-module, with $g_{k}=\left(g_{k 1}, . ., g_{k s}\right)$, then

$$
\left\{\left(e_{j}^{\prime} g_{k 1}, \ldots, e_{j}^{\prime} g_{k s}\right)\right\}_{\substack{1 \leq k \leq t \\ 1 \leq j \leq m}} \cup\left\{\left(0_{R^{m}}, \ldots, h_{l}, \ldots, 0_{R^{m}}\right)\right\}_{\substack{1 \leq i \leq s, 1 \leq l \leq r}}
$$

is a generator system of $\alpha^{-1}(T)=\alpha^{-1}\left(R^{m} \otimes_{R} B\right)+L^{s}$ as a left $R$-module, since

$$
\left\{e_{j}^{\prime} \otimes g_{k}\right\}_{\substack{1 \leq j \leq m, 1 \\ 1 \leq k \leq t}} \cup\left\{h_{l} \otimes e_{i}\right\}_{\substack{1 \leq l \leq r, 1 \leq i \leq s}}
$$

is a generator system of $T=R^{m} \otimes_{R} B+L \otimes_{R} R^{s}$ and

$$
\begin{aligned}
\alpha^{-1}(T) & =\alpha^{-1}\left(R^{m} \otimes_{R} B\right)+\alpha^{-1}\left(L \otimes_{R} R^{s}\right) \\
& =\alpha^{-1}\left(R^{m} \otimes_{R} B\right)+L^{s} \\
& ={ }_{R}\left\langle\alpha^{-1}\left(e_{j}^{\prime} \otimes g_{k}\right), \alpha^{-1}\left(h_{l} \otimes e_{i}\right)\right\rangle_{i, j, k, l} \\
& ={ }_{R}\left\langle\left(g_{k 1} e_{j}^{\prime}, \ldots, g_{k s} e_{j}^{\prime}\right),\left(0_{R^{m}}, \ldots, h_{l}, \ldots, 0_{R^{m}}\right)\right\rangle_{i, j, k, l} .
\end{aligned}
$$

\section{Computing $\operatorname{Tor}_{k}(M, N)$}

Now, let $R=k\left\{x_{1}, \ldots, x_{n} ; Q, Q^{\prime}, \preceq\right\}$ be a left PBW ring and $N$ a finitely generated left $R$-module. Let

$$
\cdots \stackrel{\partial_{k+1}}{\longrightarrow} R^{s_{k}} \stackrel{\partial_{k}}{\longrightarrow} R^{s_{k-1}} \longrightarrow \cdots \stackrel{\partial_{1}}{\longrightarrow} R^{s_{0}} \stackrel{\partial_{0}}{\longrightarrow} N \longrightarrow 0
$$

be a free resolution of $N$, where $\partial_{k}$ is the matrix

$$
\left(\begin{array}{c}
g_{1}^{k} \\
\vdots \\
g_{s_{k}}^{k}
\end{array}\right)
$$


with $g_{i}^{k}=\left(g_{i 1}^{k}, \ldots, g_{i s_{k-1}}^{k}\right) \in R^{s_{k-1}}$ for $k \geq 1$ and $1 \leq i \leq s_{k}$ (an algorithm to compute it may be found in [2, chapter 6]).

In this section we show a method for computing $\operatorname{Tor}_{k}(M, N)$ when $M$ is a finitely presented $R$-bimodule, i.e., we show how to compute the $k$-th homology module of the complex

$$
\ldots \stackrel{1_{M} \otimes \partial_{k+1}}{\longrightarrow} M \otimes_{R} R^{s_{k}} \stackrel{1_{M} \otimes \partial_{k}}{\longrightarrow} \ldots \stackrel{1_{M} \otimes \partial_{1}}{\longrightarrow} M \otimes_{R} R^{s_{0}} \longrightarrow 0 .
$$

Remark 2.1. The case $k=0$ may be treated apart, since $\operatorname{Tor}_{0}(M, N) \cong$ $M \otimes_{R} N$.

We start with the presentations $0 \rightarrow L \rightarrow R^{m} \rightarrow M \rightarrow 0$ and $0 \rightarrow$ ker $\partial_{0} \rightarrow R^{s_{0}} \rightarrow N \rightarrow 0$ of the $R$-bimodule $M$, resp. of the left $R$-module $N$.

Since $\left\{g_{1}^{1}, \ldots, g_{s_{1}}^{1}\right\}$ is a generator system of ker $\partial_{0}=\operatorname{im} \partial_{1}$ as a left $R$ module and

$$
\gamma: R^{m s_{0}} /\left(\alpha^{-1}\left(R^{m} \otimes_{R} \operatorname{ker} \partial_{0}\right)+L^{s_{0}}\right) \longrightarrow \operatorname{Tor}_{0}(M, N),
$$

as in (3) is an isomorphism of left $R$-modules, we completely describe a presentation of $\operatorname{Tor}_{0}(M, N)$ by giving the generator system

$$
\left\{\left(e_{j}^{\prime} g_{l 1}^{1}, \ldots, e_{j}^{\prime} g_{l s_{0}}^{1}\right)\right\}_{\substack{1 \leq l \leq s_{1} \\ 1 \leq j \leq m}} \cup\left\{\left(0_{R^{m}}, \ldots, h_{l}^{i}, \ldots, 0_{R^{m}}\right)\right\}_{\substack{1 \leq i \leq s_{0} \\ 1 \leq l \leq r}}^{\substack{1 \leq l \\ \text { (n) }}}
$$

of $\alpha^{-1}\left(R^{m} \otimes_{R}\right.$ ker $\left.\partial_{0}\right)+L^{s_{0}}$, where $\left\{h_{1}, \ldots, h_{r}\right\}$ is a two-sided Gröbner basis of $M$ (see (4)).

Let us return to the general case. Consider again the presentation $0 \rightarrow$ $L \rightarrow R^{m} \stackrel{p_{M}}{\rightarrow} M \rightarrow 0$ of the $R$-bimodule $M$ and the free resolution (15) of $N$.

For all $k \geq 1$, let $d_{k}=\gamma_{k-1}^{-1} \circ\left(1_{M} \otimes \partial_{k}\right) \circ \gamma_{k}$, where $\gamma_{k}: R^{m s_{k}} / L^{s_{k}} \longrightarrow$ $M \otimes_{R} R^{s_{k}}$ is the isomorphism defined as in (3) with $N=R^{s_{k}}$.

Clearly, the complex

$$
\cdots \longrightarrow R^{m s_{k}} / L^{s_{k}} \stackrel{d_{k}}{\longrightarrow} R^{m s_{k-1}} / L^{s_{k-1}} \stackrel{d_{k-1}}{\longrightarrow} \cdots \stackrel{d_{1}}{\longrightarrow} R^{m s_{0}} / L^{s_{0}} \longrightarrow 0
$$

is isomorphic to the one in (6), so $\operatorname{Tor}_{k}(M, N)$ may be computed as the homology of (7).

By definition, for all $1 \leq i \leq s_{k}, 1 \leq j \leq m$,

$$
\begin{aligned}
d_{k}\left(e_{i j}+L^{s_{k}}\right) & =\gamma_{k-1}^{-1}\left(1_{M} \otimes \partial_{k}\right) \gamma_{k}\left(e_{i j}+L^{s_{k}}\right) \\
& =\gamma_{k-1}^{-1}\left(\left(1_{M} \otimes \partial_{k}\right)\left(p_{M}\left(e_{j}^{\prime}\right) \otimes e_{i}\right)\right) \\
& =\gamma_{k-1}^{-1}\left(p_{M}\left(e_{j}^{\prime}\right) \otimes\left(g_{i 1}^{k}, \ldots, g_{i s_{k-1}}^{k}\right)\right) \\
& =\left(g_{i 1}^{k} e_{j}^{\prime}, \ldots, g_{i s_{k-1}}^{k} e_{j}^{\prime}\right)+L^{s_{k-1}} .
\end{aligned}
$$


Let $\widetilde{d}_{k}: R^{m s_{k}} \longrightarrow R^{m s_{k-1}}$ be the block-built matrix

$$
A_{k}=\left(\begin{array}{c|c|c}
g_{11}^{k} I_{m} & \cdots & g_{1 s_{k-1}}^{k} I_{m} \\
\hline \vdots & & \vdots \\
\hline g_{s_{k} 1}^{k} I_{m} & \cdots & g_{s_{k} s_{k-1}}^{k} I_{m}
\end{array}\right) \in \mathcal{M}_{m s_{k} \times m s_{k-1}}(R),
$$

where $I_{m}$ denotes the $m \times m$-identity matrix. Since $A_{k}$ is built of blocks which are elements of $R$ times the identity matrix, we have $\widetilde{d}_{k}\left(L^{s_{k}}\right) \subseteq L^{s_{k-1}}$, and the diagram

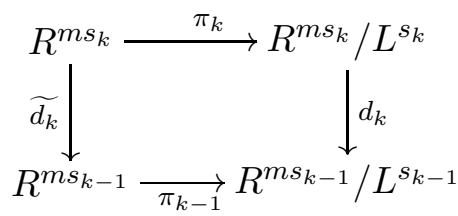

is commutative.

The above discussion proves the following result:

Theorem 2.2. With the previous notation, for all $k \geq 1$

1. $\operatorname{ker} d_{k}=\operatorname{ker} \pi_{k-1} \widetilde{d_{k}} / L^{s_{k}}$;

2. $\operatorname{im} d_{k}=\operatorname{im} \pi_{k-1} \widetilde{d}_{k} \subseteq R^{m s_{k-1}} / L^{s_{k-1}}$ is generated by

$$
\left\{\left(g_{i 1}^{k} e_{j}^{\prime}, \ldots, g_{i s_{k-1}}^{k} e_{j}^{\prime}\right)+L^{s_{k-1}}\right\}_{\substack{1 \leq i \leq s_{k} \\ 1 \leq j \leq m}}
$$

as a left $R$-module (note that $\left(g_{i 1}^{k} e_{j}^{\prime}, \ldots, g_{i s_{k-1}}^{k} e_{j}^{\prime}\right)$ is the $(j+m(i-1))$-th row of $A_{k}$ );

3. $\operatorname{Tor}_{k}(M, N) \cong \operatorname{ker} d_{k} / \operatorname{im} d_{k+1}=\operatorname{ker} \pi_{k-1} \widetilde{d}_{k} /\left({ }_{R}\left\langle\right.\right.$ rows of $\left.\left.A_{k+1}\right\rangle+L^{s_{k}}\right)$.

Remark 2.3. To compute $\operatorname{Tor}_{k}(M, N)$ we start with a finite presentation $0 \rightarrow L \rightarrow R^{m} \stackrel{p_{M}}{\rightarrow} M \rightarrow 0$ of the $R$-bimodule $M$ (say, e.g., we have computed a two-sided Gröbner basis $\left\{h_{1}, \ldots, h_{r}\right\} \subseteq R^{m}$ of the $R$ bimodule $L$ ) and a free resolution of the left $R$-module $N$ as (15).

The matrix $A_{k}$ is block-built as above, using the matrix

$$
\partial_{k}=\left(\begin{array}{c}
g_{1}^{k} \\
\vdots \\
g_{s_{k}}^{k}
\end{array}\right)
$$

The set $\left\{\left(0_{R^{m}}, \ldots, h_{l}^{i}, \ldots, 0_{R^{m}}\right)\right\}_{i, l=1}^{s_{k}, r}$ is a generator system (in fact, it is a two-sided Gröbner basis when $\left\{h_{1}, \ldots, h_{r}\right\}$ so is) of $L^{s_{k}}$ as an $R$-bimodule. 
Then, we compute the kernel of $\pi_{k-1} \widetilde{d_{k}}: R^{m s_{k}} \longrightarrow R^{m s_{k-1}} / L^{s_{k-1}}$ using syzygies. Indeed (see [1] ), let $H_{k}$ be the matrix

$$
H_{k}=\left(\frac{A_{k}}{A_{k}^{\prime}}\right) \in \mathcal{M}_{\left(m s_{k}+r s_{k-1}\right) \times m s_{k-1}}(R),
$$

where $A_{k}^{\prime}$ is the matrix whose rows are the generators of $L^{s_{k-1}}$ as a left $R$-module. Then, if

$$
\operatorname{Syz} z^{l}\left(H_{k}\right)={ }_{R}\left\langle p_{1}, \ldots, p_{l}\right\rangle, \quad \text { with } p_{i}=\left(p_{i}^{\prime}, p_{i}^{\prime \prime}\right) \in R^{m s_{k}} \times R^{r s_{k-1}},
$$

then $\operatorname{ker} \pi_{k-1} \widetilde{d}_{k}={ }_{R}\left\langle p_{1}^{\prime}, \ldots, p_{l}^{\prime}\right\rangle$.

Example 2.4. Let $R=U(\mathfrak{s l}(2))$, the universal enveloping algebra of the Lie algebra of traceless $2 \times 2$-matrices, where $k=\mathbb{C}($ or $k=\mathbb{Q})$. We know (see, e.g., [2]) that $R$ is the PBW algebra $k\{x, y, z ; Q \preceq \omega\}$ with $Q=$ $\{y x-x y+z, z x-x z-2 x, z y-y z+2 y\}$ and, say, $\omega=(1,2,2)$.

Let $N=R^{2} / B$, where $B$ is the left $R$-module generated by

$$
g_{1}=\left(y^{3}, x\right), g_{2}=(y, x z), g_{3}=\left(0, x y^{2} z-2 y z^{2}+2 y z-x\right)
$$

The left syzygy module $\operatorname{Syz}^{l}\left(g_{1}, g_{2}, g_{3}\right)$ is generated by $g=\left(1,-y^{2}, 1\right) \in R^{3}$, and hence

$$
0 \longrightarrow R \stackrel{\delta_{1}}{\longrightarrow} R^{3} \stackrel{\delta_{0}}{\longrightarrow} N \longrightarrow 0
$$

where $\partial_{0}=\left(\begin{array}{c}g_{1} \\ g_{2} \\ g_{3}\end{array}\right)$ and $\partial_{1}=(g)$, is a free resolution of $N$.

Let $L$ be the $R$-bimodule generated by $\{(C, 1),(1, C)\}$, where $C$ is the Casimir element $z^{2} / 2+2 x y-z$ (a well known central element of $\left.U(\mathfrak{s l}(2))\right)$, and let $M=R^{2} / L$.

For all $k \geq 2$ we have $\operatorname{Tor}_{k}(M, N)=0$, as the free resolution (9) of $N$ has length 2.

For $k=0$, we have (see 2.1) $\operatorname{Tor}_{0}(M, N) \cong R^{6} /\left(\alpha^{-1}\left(R^{2} \otimes_{R} B\right)+L^{3}\right)$, and

$$
\begin{gathered}
\left\{\left(1,0,-y^{2}, 0,1,0\right),\left(0,1,0,-y^{2}, 0,1\right),(C, 1,0,0,0,0),(0,0, C, 1,0,0)\right. \\
(0,0,0,0, C, 1),(1, C, 0,0,0,0),(0,0,1, C, 0,0),(0,0,0,0,1, C)\}
\end{gathered}
$$

is a generator system of $\alpha^{-1}\left(R^{2} \otimes_{R} B\right)+L^{3}$ as a left $R$-module.

For $k=1$, we have $\operatorname{Tor}_{1}(M, N) \cong \operatorname{ker}\left(\pi_{0} \widetilde{d_{1}}\right) /\left({ }_{R}\left\langle\right.\right.$ rows of $\left.\left.A_{2}\right\rangle+L\right)$, but in this particular example $A_{2}=0$ since $\partial_{2}=0$. 
As pointed out in 2.3, $\operatorname{ker} \pi_{0} \widetilde{d}_{1}$ is obtained from the left syzygy module of the rows of

$$
A_{1}=\left(\begin{array}{cccccc}
1 & 0 & -y^{2} & 0 & 1 & 0 \\
0 & 1 & 0 & -y^{2} & 0 & 1
\end{array}\right)
$$

and the generators of $L^{3}$. Indeed, by picking up just the first two components of each element of the generator system

$$
\begin{aligned}
& \left\{\left(z^{2}+4 x y-2 z, 2,-2,2 y^{2},-2,0,0,0\right)\right. \\
& \left(1, z^{2} / 2+2 x y-z, 0,0,0,-1, y^{2},-1\right) \\
& \left(8 x y-4 z,-4 z^{4}-4 x y z^{2}+2 z^{3}+4,-4,4 y^{2},-4,2 y^{2},\right. \\
& \left.\left.\quad-2 y^{2} z^{2}+16 y^{2} z-32 y^{2}, 2 z^{2}\right)\right\}
\end{aligned}
$$

of the left syzygy module, we obtain the generator system

$$
\left\{(2 C, 2),(1, C),\left(8 x y-4 z,-z^{4}-4 x y z^{2}+2 z^{3}+4\right)\right\}
$$

of $\operatorname{ker} \pi_{0} \widetilde{d}_{1}$. Therefore, $\operatorname{Tor}_{1}(M, N) \cong \operatorname{ker} \pi_{0} \widetilde{d}_{1} / L=0$, since $\left(8 x y-4 z,-z^{4}-\right.$ $\left.4 x y z^{2}+2 z^{3}+4\right) \in L$ (one may check this out by dividing the element by a two-sided Gröbner basis of $L$ ).

\section{Acknowledgement}

Both authors would like to thank Pepe Bueso for his useful suggestions.

\section{References}

[1] Bueso, J.L.; Castro, F.; Gómez Torrecillas, J.; Lobillo, F.J. Homological computations in PBW modules. Alg. Rep. Theory 2001, 4 (2), 201-218.

[2] Bueso, J.L.; Gomez Torrecillas, J.; Verschoren, A. Algorithmic Methods in Noncommutative Algebra: Applications to Quantum Groups; Kluwer: Dordrecht, The Netherlands, 2003.

[3] García Román, S.; García Román, M. Gröbner bases and syzygies on bimodules, preprint. math.RA/0405550

[4] Smith, G. Computing global extension modules. J. Symb. Comput. 2000, 29 (4-5), 729-746. 